\title{
Onset of oscillatory binary fluid convection in three-dimensional cells
}

\author{
Arantxa Alonso \\ Departament de Física Aplicada, Universitat Politècnica de Catalunya, \\ Campus Nord, Mòdul B4, 08034 Barcelona, SPAIN \\ arantxa@fa.upc.es \\ Oriol Batiste \\ Departament de Física Aplicada, Universitat Politècnica de Catalunya, \\ Campus Nord, Mòdul B4, 08034 Barcelona, SPAIN \\ oriol@fa.upc.es \\ Communicated by
}

Received date and accepted date

\begin{abstract}
The purpose of this work is to investigate the influence of the transverse walls on the onset of convection in a horizontal rectangular cavity of infinite length filled with a binary mixture when heated from below. For the first time we take into account the effect of the third dimension without making any approximation and considering realistic boundary conditions. In previous numerical works the width of the cell was either taken to be infinity (bulk mixtures) or different approximations usually valid in the narrow cell limit were assumed (i.e. Hele-Shaw and non-ideal Hele-Shaw approximations). The results we find show that the presence of the walls has a considerable effect on the onset of convection even for intermediate transverse aspect ratio cells. They also show that the approximations generally assumed fail to reproduce the correct behaviour of the critical parameters in an important range of aspect ratio values when the primary bifurcation is oscillatory. We have compared the critical values of the Rayleigh number and the frequency that we obtain with those reported in the literature (Ohlsen et al., 1990) and we find a quantitative agreement within the experimental error.
\end{abstract}

\section{Introduction}

Thermal convection in binary fluids mixtures has attracted much research activity in the past and has become a paradigmatic experimental system for the study of nonlinear wave phenomena and pattern formation (Cross and Hohenberg, 1993). In comparison to the pure fluid case, the dynamics is more complicated due to the extra degree of freedom associated with the concentration field. In a pure fluid a temperature gradient produces a density gradient and the fluid becomes potentially unstable in the presence of gravity. In a binary mixture, the temperature gradient also produces a flux of mass. This is denoted as the Soret effect. The density gradient is thus modified by the concentration gradient induced by the Soret effect. The nondimensional parameter that quantifies the influence of this effect on the convective buoyancy force is the separation ratio $S$. When $S>0$ the denser component tends to move towards the colder region. The threshold of instability is dramatically lowered, but the arising convective pattern remains stationary. If $S<0$ the denser component 
migrates towards the hotter region, with the Soret diffusion having a stabilizing effect on the fluid layer. In this case the first instability of the conduction state is already oscillatory, fact that makes this system very interesting from the point of view of dynamical systems because it leads to a rich dynamical behaviour near onset of convection. The oscillatory nature of the instability in negative separation ratio binary mixtures is argued to be realated to the competition of two diffusion processes (diffusion of heat and mass), which act on very different timescales (Bensimon et al., 1990). Near threshold, when a fluid element is displaced vertically in the presence of vertical temperature and concentration gradients, it reaches thermal equilibrium very fast, but it retains a difference in concentration with its new surroundings on the timescale of typical fluid motions. Therefore, the element feels a restoring force caused by the resulting density difference and behaves like a relaxation oscillator.

Experiments on binary mixtures are frequently made using different types of solutions of ethanol in water, for which the value of the separation ratio can range typically from -0.5 to +0.5 , although other types of solutions, such as mixtures of ${ }^{3} \mathrm{He}-{ }^{4} \mathrm{He}$ with a separation ratio of around -0.02 have also been used. When the specific weights of the two components are quite distinct, such in the case of ferrofluids (dispersions of magnetite in water or oil), the binary mixture is known to exhibit a large value of the separation ratio $(|S| \sim 10$ or even larger). In this paper we will focus on binary mixtures with negative values of the separation ratio.

Although the evolution equations that describe this system are well known and validated, and have been used for the derivation of amplitude equation models and in full numerical simulations that compare very well with experiments (Batiste et al., 2001), the available numerical computations do not take into account the effect of the transverse walls in an exact way. In some models, the features of binary convection near the onset (critical temperature difference, wavenumber and frequency) have been obtained neglecting the effect of transverse walls, both in infinite extended horizontal layers often referred to as 'bulk binary mixtures' (Knobloch and Moore, 1988; Cross and Kim, 1988), and in laterally bounded domains (Batiste et al., 1999). The results obtained with these two-dimensional models are correct for wide enough containers. Other computations reproduce convection in very narrow cells (Hele-Shaw cells) or include approximations such as the non-ideal Hele-Shaw approximation (Schöpf, 1992) that try to model the presence of the transverse walls in intermediate-width cells. At present, as far as the authors know, there are no published quantitative predictions in which the three-dimensional problem is solved exactly even in the linear case.

Nevertheless, the presence of the transverse walls is expected to play an important role in experimental binary convection. Most of the experiments on binary mixtures have been made using narrow convection cells in order to suppress three-dimensional instabilities and to avoid the formation of spatial defects in the rolls system. Some of them use long rectangular cells with horizontal aspect ratios (length to height ratio) typically ranging from 20 to 40 , but with transverse aspect ratios (width to height ratio) varying from 3 to 4 (Lhost and Platten, 1989; Kolodner, 1993), being sometimes even narrower, 0.3 and 0.5 (Liu and de Bruyn, 1992; Schöpf and Rehberg, 1992). Other experimental setups use long annular containers with large horizontal aspect ratios (mean circumference length to height ratio) of about 70, but again they use small transverse aspect ratio cells of 1.28 and 1.63 (Ohlsen et al., 1990; Bensimon et al., 1990) in which the effect of the friction exerted by the walls should be significant. These experiments report discrepancies with the available numerical predictions both in the onset of convection and in the nonlinear regime.

In this work we present a linear stability analysis of the basic state for a binary fluid mixture contained in a narrow three-dimensional rectangular cell. We will solve the full three-dimensional equations with periodic boundary conditions in the long dimension and experimental boundary conditions in the other walls. The periodic boundary conditions we assume in the horizontal direction model annular containers (the use of cartesian coordinates is justified due to the small curvature of the cells used in experiments), but the values of the critical parameters are also correct for sufficiently large rectangular cells. The aim of our work is to investigate the influence of the transverse walls on the onset of convection and to check the validity of the approximations usually made. 


\section{Governing Equations and Numerical Method}

We consider a box of infinite horizontal length ( $x$-direction) with height $d$ (z-direction) and width $b$ (ydirection) filled with a Boussinesq binary fluid of thermal and mass diffusivities $\kappa$ and $D$, kinematic viscosity $\nu$, thermal and concentration expansion coefficients $\alpha$ and $\beta$, and Soret coefficient $S_{T}$. The box is heated from below in the presence of a vertical gravity $\mathbf{g}=-g \hat{\mathbf{e}}_{z}$. There exists a basic stationary conduction state with vertical temperature and concentration gradients,

$$
\begin{gathered}
\mathbf{u}_{c}=0, \\
T_{c}=T_{0}-\Delta T\left(\frac{z}{d}-\frac{1}{2}\right), \\
C_{c}=C_{0}+C_{0}\left(1-C_{0}\right) S_{T} \Delta T\left(\frac{z}{d}-\frac{1}{2}\right),
\end{gathered}
$$

with $\Delta T$ being the imposed temperature difference and $T_{0}$ and $C_{0}$ the mean temperature and concentration. The dynamics of this system is governed by the Navier-Stokes, continuity, energy and mass conservation equations which, once nondimensionalized by using the height of the layer $d$ as lengthscale, $d^{2} / \kappa$ as timescale and $\Delta T$ as temperature scale, take the form

$$
\begin{gathered}
\partial_{t} \mathbf{u}+(\mathbf{u} \cdot \nabla) \mathbf{u}=-\nabla p+\sigma \nabla^{2} \mathbf{u}+[R a(1+S) \Theta+\sigma R a S \eta] \hat{\mathbf{e}}_{z}, \\
\nabla \cdot \mathbf{u}=0, \\
\partial_{t} \Theta+(\mathbf{u} \cdot \nabla) \Theta=u_{z}+\nabla^{2} T, \\
\partial_{t} \eta+(\mathbf{u} \cdot \nabla) \eta=-\nabla^{2} \Theta+\tau \nabla^{2} \eta .
\end{gathered}
$$

Here, $\mathbf{u}=\left(u_{x}, u_{y}, u_{z}\right)$ is the velocity field, $\Theta$ denotes the departure of the temperature from its conduction profile, $T=\Theta+T_{c}$, and $\eta=C-C_{c}-\Theta$, where $C$ is the concentration of the denser component. The Rayleigh, Prandtl and Lewis numbers and the separation ratio are defined by

$$
R a=\frac{\alpha \Delta T g d^{3}}{\kappa \nu}, \quad \sigma=\frac{\nu}{\kappa}, \quad \tau=\frac{D}{\kappa}, \quad S=C_{0}\left(1-C_{0}\right) \frac{\beta}{\alpha} S_{T},
$$

and together with the transverse aspect ratio, $\Gamma_{y}=b / d$, form the set of nondimensional numbers that describe the system. The boundary conditions adopted will be those relevant to experiments. We have considered periodic boundary conditions in the $\mathrm{x}$ direction (annular containers), no-slip boundary conditions for the velocity in the solid walls, perfectly conducting top and bottom walls, insulating front and back walls and we we have imposed the requirement that there is no mass flux through any of the boundaries

$$
\begin{array}{lr}
\mathbf{u}=\mathbf{n} \cdot \nabla \eta=0 & \text { on } \partial \Omega, \\
\Theta=0 & \text { on } z=0,1, \\
\partial_{y} \Theta=0 & \text { on } y=0, \Gamma_{y} .
\end{array}
$$

The linearized equations describing the stability of the conduction state have been solved numerically using a formulation based on velocity potentials (Goldstein et al., 1993) in which the velocity field is written as

$$
\mathbf{u}=\nabla \times\left(\xi \hat{\mathbf{e}}_{y}+\Psi \hat{\mathbf{e}}_{z}\right)
$$

We look for solutions of the form

$$
\Phi(x, y, z, t)=\hat{\Phi}(x, y, z) e^{(s+i \omega) t},
$$

and we reach an eigenvalue problem of the type $A \Phi=(s+i \omega) B \Phi$, where

$$
A=\left(\begin{array}{cccc}
-\sigma \nabla^{2}\left(\partial_{x x}^{2}+\partial_{y y}^{2}\right) & \sigma \nabla^{2} \partial_{y z}^{2} & 0 & 0 \\
\sigma \nabla^{2} \partial_{y z}^{2} & -\sigma \nabla^{2}\left(\partial_{x x}^{2}+\partial_{z z}^{2}\right) & -\sigma R a(1+S) \partial_{x} & -\sigma R a S \partial_{x} \\
0 & \partial_{x} & \nabla^{2} & 0 \\
0 & 0 & -\nabla^{2} & \tau \nabla^{2}
\end{array}\right)
$$




$$
B=\left(\begin{array}{cccc}
-\left(\partial_{x x}^{2}+\partial_{y y}^{2}\right) & \partial_{y z}^{2} & 0 & 0 \\
\partial_{y z}^{2} & -\left(\partial_{x x}^{2}+\partial_{z z}^{2}\right) & 0 & 0 \\
0 & 0 & 1 & 0 \\
0 & 0 & 0 & 1
\end{array}\right), \quad \Phi=\left(\begin{array}{c}
\Psi \\
\xi \\
\Theta \\
\eta
\end{array}\right)
$$

To solve the eigenvalue problem we use a pseudospectral collocation method. The spatial dependence of the variables has been expanded in terms of basis $A_{l}(y)$ and $B_{m}(z)$ for the $y$ and $z$ dependence, which are combinations of the Chebyshev polynomials that satisfy the boundary conditions, and Fourier expansions for the periodic direction

$$
\hat{\Phi}(x, y, z)=e^{i k x} \sum_{l, m} \alpha_{k l m} A_{l}(y) B_{m}(z),
$$

being $k$ the horizontal wavenumber of the pattern. The condition $s=0$ defines the onset of convection in the system. We need to solve the eigenvalue problem for each set of the parameter values, $\left(\sigma, \tau, S, \Gamma_{y}\right)$. Then, by minimizing the Rayleigh number with respect to the wavenumber $k$, we find the critical values of the parameters (Rayleigh, frequency and wavenumber) and the spatial dependence of the bifurcating modes, which is given by the eigenfunctions. In order to decrease the size of the eigenvalue problem we have taken into account the parity in $y$ and $z$ of the functions.

\section{Results}

In this section we will present the results obtained for a water-ethanol mixture and we will compare them to the analogous results for a pure fluid. We have chosen as reference values for our computations the parameters of the fluid and the geometry of the container presented in Ohlsen et al. (1990). The cell they consider is a long, narrow annulus with a transverse aspect ratio $\Gamma_{y}=1.288$ and a horizontal aspect ratio $\Gamma_{x}=67.09$. The binary fluid is a water-ethanol mixture with $\sigma=9.16, \tau=0.008$ and $S=-0.257$. The transverse walls of the container are made of plastic, so adiabatic boundary conditions on these walls are the correct choice to model this experiment. All the results reported in this paper correspond to $\sigma=9.16$ and $\tau=0.008$.

\subsection{Water-ethanol mixture}

We will describe first the neutral stability curves of the conduction state for a water-ethanol mixture. We have analyzed the dependence of the critical parameters both with the transverse aspect ratio of the container and with the separation ratio of the mixture. Then we will discuss the structure of the arising patterns of convection.

In figure 1 we explore the dependence of the critical parameters (Rayleigh number, frequency and wavenumber) on the transverse aspect ratio of the container and compare the results we obtain by solving the exact three-dimensional stability problem (thick solid line) with those resulting from the non-ideal Hele-Shaw approximation (thin solid line), from the Hele-Shaw limit (dashed line) and from a bulk mixture (dashed-dotted line). In the Hele-Shaw approximation the term $\nabla^{2} \mathbf{u}$ in the Navier-Stokes equation is replaced by $-12 \mathbf{u} / \Gamma_{y}^{2}$, after assuming a parabolic profile for the velocity, neglecting the transverse velocity component and considering very narrow cells. In the non-ideal Hele-Shaw approximation the effect of finite width is taken into account by adding the viscosity term resulting from the the Hele-Shaw approximation to the laplacian in the two-dimensional problem (infinite width cell). Thus the laplacian in the viscosity term is replaced by $\nabla^{2}=\partial_{x}^{2}+\partial_{z}^{2}-12 / \Gamma_{y}^{2}$ (Schöpf, 1992). As expected, we can see in the plots included in figure 1 that the critical values resulting from the Hele-Shaw approximation are only correct for extremely narrow cells. If the aspect ratio is slightly increased the results become totally wrong. We also see that both the curves corresponding to the non-ideal Hele-Shaw approximation and to the 3D computation reproduce correctly the Hele-Shaw limit (very narrow cells) and the bulk mixture limit (infinite aspect ratio cells). Surprisingly, the plots show that for a wide range of intermediate aspect ratio values the results obtained with the non-ideal Hele-Shaw approximation do not agree with those resulting from the 3D computation. We find discrepancies in the critical Rayleigh number as well as in the dependence of the critical wavenumber 
and frequency on the aspect ratio. As it is well-known, the friction of the transverse walls on the fluid results in an increase of the critical Rayleigh number, but our results show that the non-ideal Hele-Shaw approximation underestimates this effect. As far as the behaviour of the critical wavenumber is concerned, the neutral stability curves for the selected wavenumber of the pattern present both a maximum, but its position and value is wrongly predicted by the non-ideal Hele-Shaw model. While this approximation finds the maximum for $\Gamma_{y} \approx 0.3$, the 3D linear stability analysis locates it in $\Gamma_{y} \approx 2$. It is also worth emphasizing that the differences between the $3 \mathrm{D}$ and the $2 \mathrm{D}$ computations extend for a much wider range of aspect ratio values than would be expected from the non-ideal Hele-Shaw results.

\begin{tabular}{|l|c|c|c|}
\hline & $R a_{c}$ & $k_{c}$ & $\omega_{c}$ \\
\hline \hline Experiment & $3074 \pm 17$ & 3.278 & 13.8 \\
\hline 2D Computation & 2292 & 3.137 & 11.4 \\
\hline Non-ideal Hele-Shaw & 2726 & 3.16 & 11.5 \\
\hline 3D Computation & 3063 & 3.35 & 13.7 \\
\hline 3D Computation $\left(k_{e x p}\right)$ & 3064 & 3.278 & 13.4 \\
\hline
\end{tabular}

Table 1. Experimental (Ohlsen et al., 1990) and numerically computed values of the critical parameters for a binary mixture with $S=-0.257$ contained in a cell of transverse aspect ratio $\Gamma_{y}=1.288$.
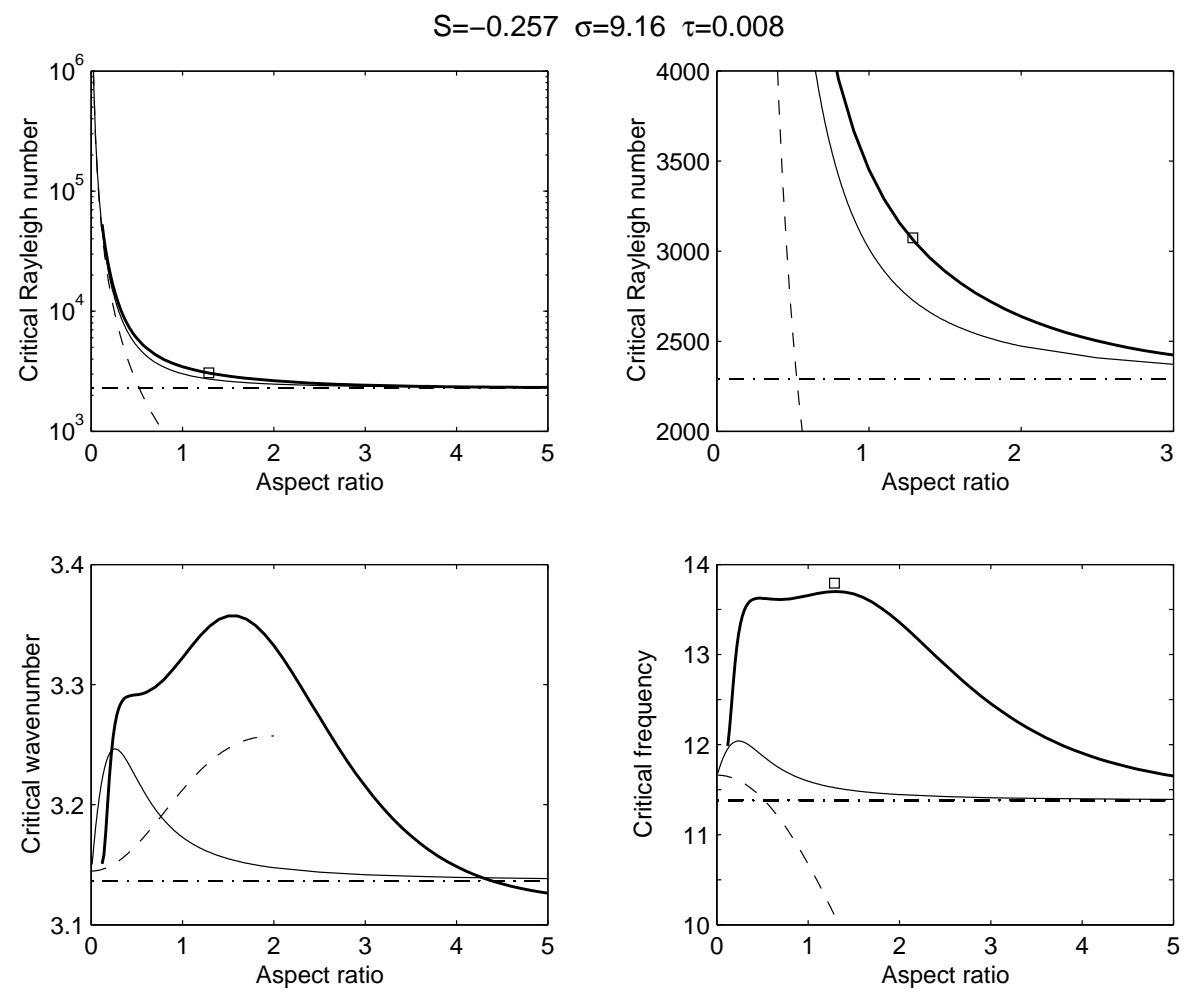

Figure 1. Comparison of the neutral stability curves (critical Rayleigh number, frequency and wavenumber as a function of the transverse aspect ratio) obtained by solving the 3D stability problem (thick solid line) and by using the non-ideal Hele-Shaw (thin solid line) and the Hele-Shaw (dashed line) approximations for a binary mixture with $S=-0.257$. The dashed-dotted line shows the critical Rayleigh number for the bulk mixture and the square the one found in the experiment reported in Ohlsen et al. (1990). 

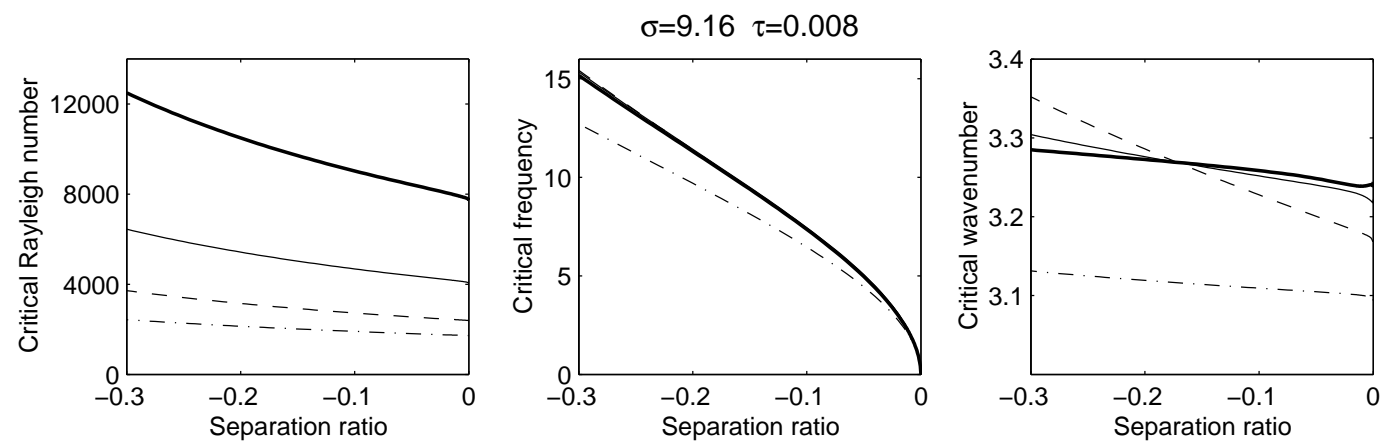

Figure 2. Neutral stability curves (critical Rayleigh number, frequency and wavenumber as a function of the separation ratio) for rectangular cells with transverse aspect ratios $\Gamma_{y}=10$ (dashed-dotted line), $\Gamma_{y}=1$ (dashed line), $\Gamma_{y}=0.5$ (solid line) and $\Gamma_{y}=0.3$ (thick solid line).

The comparison with the experimental values presented in Ohlsen et al. (1990) (a square in the plots) shows an excellent agreement between the 3D calculations and the experiment. The numerical values obtained in the different cases are included in table 1. Experimentally, the reported value of the critical Rayleigh number is $R a_{c}^{\exp }=3074$, with an error $\Delta R a_{c}^{\exp }=17$, with the selected wavenumber $k_{c}^{\exp }=3.278$. In a bulk mixture theory predicts onset of convection at $R a_{c}^{\mathrm{b}}=2292$ at the critical wavenumber $k_{c}^{\mathrm{b}}=3.137$, which is rather far from the experimental values. The predictions are slightly improved when the non-ideal Hele-Shaw approximation is considered, as it gives $R a_{c}^{\text {niHs }}=2726$ for $k_{c}^{\text {niHs }}=3.16$, but the Rayleigh number is still $\approx 12 \%$ smaller than observed. Finally, the 3D stability analysis of the conduction state sets the onset of convection at a critical Rayleigh number $R a_{c}^{3 \mathrm{D}}=3063$, which falls within the experimental error, with a selected wavenumber $k_{c}^{3 \mathrm{D}}=3.35$. The 3D computations also improve drastically the predicted values of the Hopf frequency. While the experimental critical frequency of the pattern is found to be $\omega_{c}^{\exp }=13.8$, the approximations that might be suitable in this case give smaller and very similar values for this parameter. The value obtained in the non-ideal Hele-Shaw case is $\omega_{c}^{\text {nirs }}=11.5$ and that of a bulk mixture $\omega_{c}^{\mathrm{b}}=11.4$. When the $3 \mathrm{D}$ problem is solved the predicted value raises to $\omega_{c}^{3 \mathrm{D}}=13.7$. So the agreement with the experimental results is excellent, although further comparison with other experiments would be of interest and will try to be made.

In figure 2 the critical Rayleigh number, critical frequency and the selected wavenumber are plotted as a function of the separation ratio for four values of the aspect ratio, $\Gamma_{y}=10,1,0.5,0.3$. As expected, for negative separation ratio mixtures, the critical Rayleigh number increases when the separation ratio decreases. The effect of finite width of the cell on the critical Rayleigh number is important for low aspect ratios. For $\Gamma_{y}=0.5$ and $\Gamma_{y}=0.3$ the Rayleigh number is about 3 and 4 times larger, respectively, than in the 2D case due to the friction of the walls. For $\Gamma_{y}=1$ the differences are smaller, but they still can be of about $30 \%$. The effect of the width of the cell on the frequency of the pattern seems to be more important as the separation ratio of the fluid decreases. The three curves corresponding to the small aspect ratio containers lie together above the one that would correspond to a bulk mixture. Finally, it can be inferred from the last plot in figure 2 that the influence of the separation ratio on the selected wavenumber of the pattern is much more important for low values of the aspect ratio. While for $\Gamma_{y}=10$ the wavenumber is nearly independent of the separation ratio, for $\Gamma_{y}=0.3$ it varies from about $k_{c}=3.17$ to $k_{c}=3.37$. The curve for the $\Gamma_{y}=10$ container lies substantially below those for the small width cells. This fact indicates that the presence of the transverse walls would shorten the wavelength of the selected pattern at onset of convection.

The study of the onset of convection has been completed with the examination of the spatial structure of the arising convective patterns. The primary Hopf bifurcation gives rise to a time-dependent pattern. The two-dimensional linear stability analysis of the conduction state (Knobloch and Moore, 1988; Cross and Kim, 1988) shows that two branches of solutions bifurcate at the same time: solutions in the form of travelling waves and standing waves. It is also known from experiments and nonlinear numerical simulations that the primary bifurcation is subcritical and gives rise to unstable patterns. The features of these timedependent solutions are well-known in the 2D case (infinite-width cell). The travelling waves are only 
possible in unbounded or periodic domains in the horizontal direction. They travel in this direction, so the pattern breaks both the translation and reflection symmetry in the horizontal direction. The standing waves are superpositions of two oppositely travelling waves of equal amplitude. The resulting pattern breaks the translation symmetry but keeps the reflection symmetry of the system. In the case of annular convection the solutions observed in the experiments are the travelling waves, so we will restrict ourselves to the analysis of the eigenfunctions corresponding to the three-dimensional travelling waves. Our aim is to investigate how the pattern is modified when the third dimension is included. We seek a physical interpretation that accounts for the discrepancies described above between the 3D results and the different approximations.

To visualize the spatial structure of the travelling wave we have plotted the contour plots for the velocity, temperature and concentration fields in several planes. All the plots correspond to a binary mixture with $S=-0.257$ and $\Gamma_{y}=1.288$. For the sake of clearness, a representation of the geometry of the system is included on the left part of figure 3. To analyze the effect of the transverse direction we need to plot the contour plots in the XY-plane (horizontal plane) and in the YZ-plane (transverse plane). The right part of figure 3 shows a top view of the travelling wave (XY-plane). The different fields are out of phase, as a result of the pattern being travelling in the horizontal direction. The reflection symmetry in the $\mathrm{x}$-direction is clearly broken, as it is evident from the contour plots of the vertical component of the velocity, temperature and concentration. The friction of the transverse walls with the fluid deforms the pattern. The cell leads in the middle and lags close to the boundaries and, for an intermediate transverse aspect ratio like the one considered here, $\Gamma_{y}=1.288$, the pattern clearly departs from two-dimensionality.

Figure 4 shows a side view of the travelling wave (YZ-plane). The contour plots in the YZ-plane have been depicted in five time-instants to visualize the evolution of the pattern in half a period. In the non-ideal Hele-Shaw approximation a parabolic profile for the velocity is assumed and the transverse velocity $u_{y}$ is neglected. To see if this approximation is justified, since we are not including the value of the fields in the contour plots, we have also plotted in figure 4 the three components of the velocity field for a fixed value of the z-coordinate in order to compare their relative values. We see that although $u_{y}$ is generally smaller than the other two components, $u_{x}$ and $u_{z}$ (see, for instance, $t=0.1 T$ ), it is not always negligible in front of them. In the time-instant $t=0.2 T, u_{z}$ is only about two times larger than $u_{y}$, and $u_{x}$ about three times larger. Apart from that, the contour plot of the vertical velocity component $u_{z}$ shows that the travelling wave develops a non-trivial structure in the YZ-plane. In half a period $u_{z}$ changes its sign. But instead of doing this process simply decreasing its value and keeping the same structure, like the other velocity components, $u_{x}$ and $u_{y}$, the roll seems to split into two. The same process can be observed in the temperature and concentration fields. This non-trivial structure in the YZ-plane would mean that there is a departure from the expected parabolic profile. This fact is being skipped in the non-ideal Hele-Shaw approximation, and
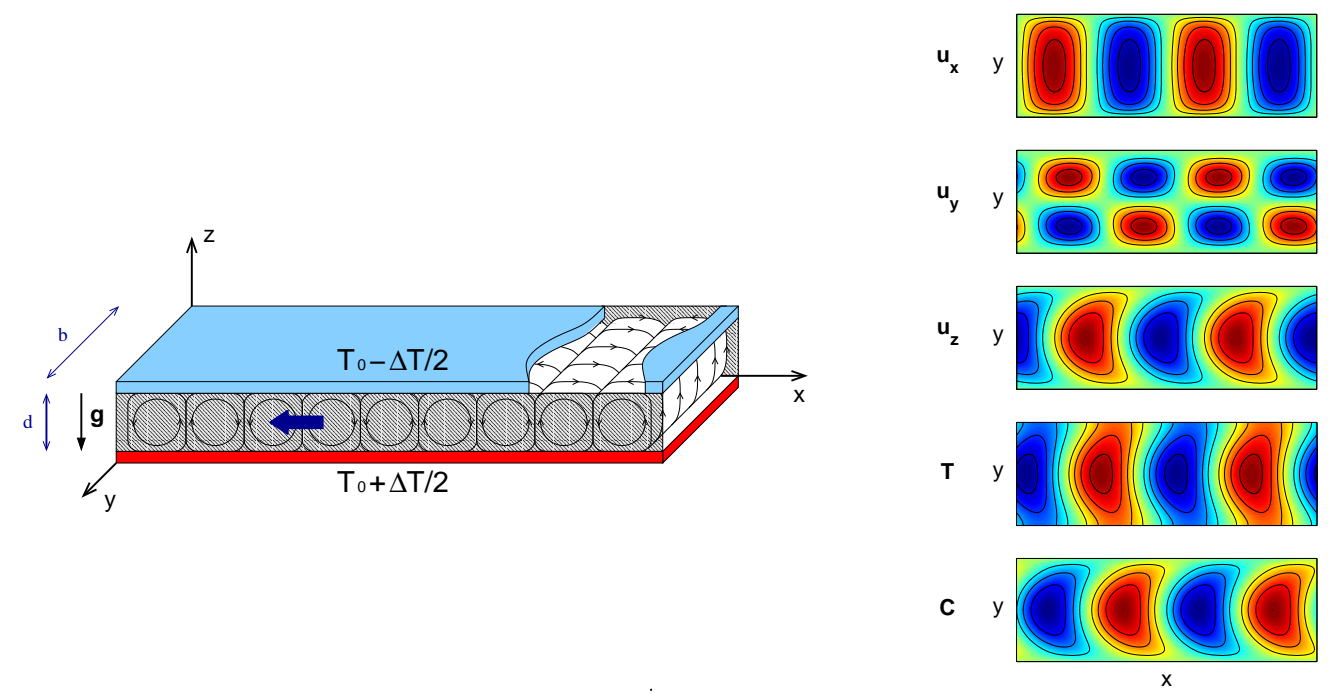

Figure 3. 3D travelling waves: top view. On the left, representation of the geometry of the system we are studying. On the right, contour plots of the velocity, temperature and concentration fields showing the structure of the 3D travelling wave in a horizontal plane. 


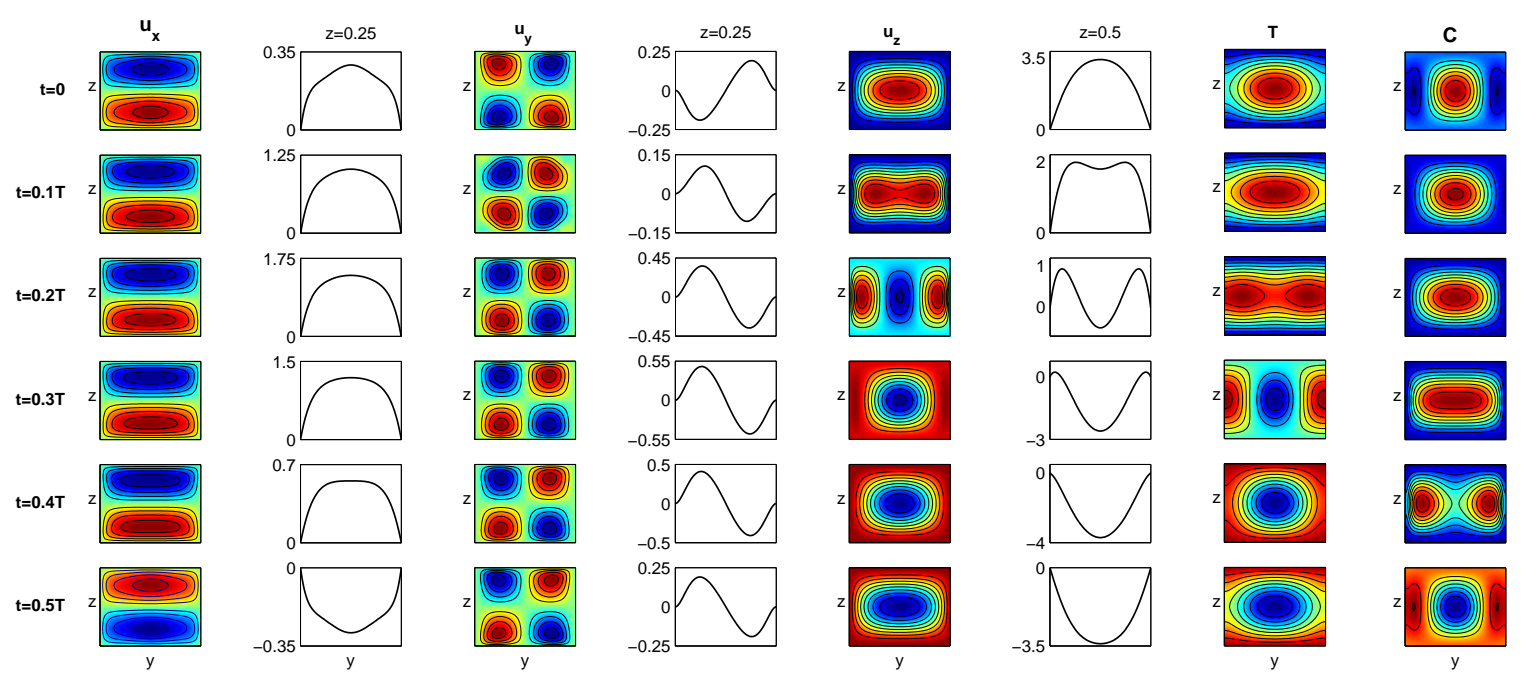

Figure 4. 3D travelling waves: side view. Contour plots of the velocity, temperature and concentration fields in five time-instants showing the structure of the 3D travelling wave in a transverse plane and its evolution in half a period and plots of the velocity components $\left(u_{x}, u_{y}\right.$ and $\left.u_{z}\right)$ showing their variation with $y$ for a fixed value of $z$.

that would explain the discrepancies between the values of the critical parameter it predicts and the real ones. This would also explain that the differences between the 3D and 2D computations extend for a wider range of intermediate aspect ratios than might be thought beforehand.

\subsection{Pure fluid}

The analogous neutral stability curves showing the dependence of the critical Rayleigh number on the transverse aspect ratio for a pure fluid $(S=0)$ have been included in figure 5 . We observe in the plot that the differences in the critical Rayleigh number between the 3D computation and the non-ideal Hele-Shaw approximation are considerably smaller than for a binary mixture. Indeed this approximation reproduces quite well the behaviour of the critical parameter except for a small region of intermediate aspect ratio values. As can be seen in table 2, the greatest differences between the critical Rayleigh number resulting from the non-ideal Hele-Shaw approximation and the one given by the 3D computation for a pure fluid are of about $6 \%$, while they are of about $15 \%$ for a binary mixture. We also see that in the case of a pure fluid the curve obtained with the exact 3D computation tends faster to that of the 2D problem. While for $\Gamma_{y}=2$ the discrepancies between the two values of the critical Rayleigh number in the case of a binary mixture are of about $13 \%$ they are of about $7 \%$ in a pure fluid (table 2 ).

In the case of a pure fluid the primary bifurcation is stationary. The contour plots showing the structure of the convective 3D steady-state pattern that arises in a $\Gamma_{y}=1.288$ container have been included in figure 6 . The left part of the figure corresponds to a top view (XY-plane) of the solution. Unlike the travelling waves, this solution keeps the reflection symmetry in the horizontal direction. The contour plots in the middle of figure 6 correspond to a side view (YZ-plane) of the pattern. The transverse aspect ratio of the cell is small, so the friction exerted by the walls forces the solution to be clearly three-dimensional and delays the onset of convection. But an inspection of the variation of the velocity components in the transverse direction $y$ for a fixed value of the vertical coordinate $z$ (right part of figure 6) shows that the transverse velocity component $u_{y}$ is considerably smaller than the other two components $\left(u_{x} \approx 3.5 u_{y}\right.$ and $\left.u_{z} \approx 10 u_{y}\right)$. This, together with the fact that now the vertical velocity component $u_{z}$ exhibits a one-roll structure, makes the velocity profile remain essentially parabolic. So the assumptions made in the non-ideal Hele-Shaw approximation, as we see, are fulfilled in the case of the steady-state pattern in pure fluid convection but they are less justified in the case of oscillatory binary mixture convection. 

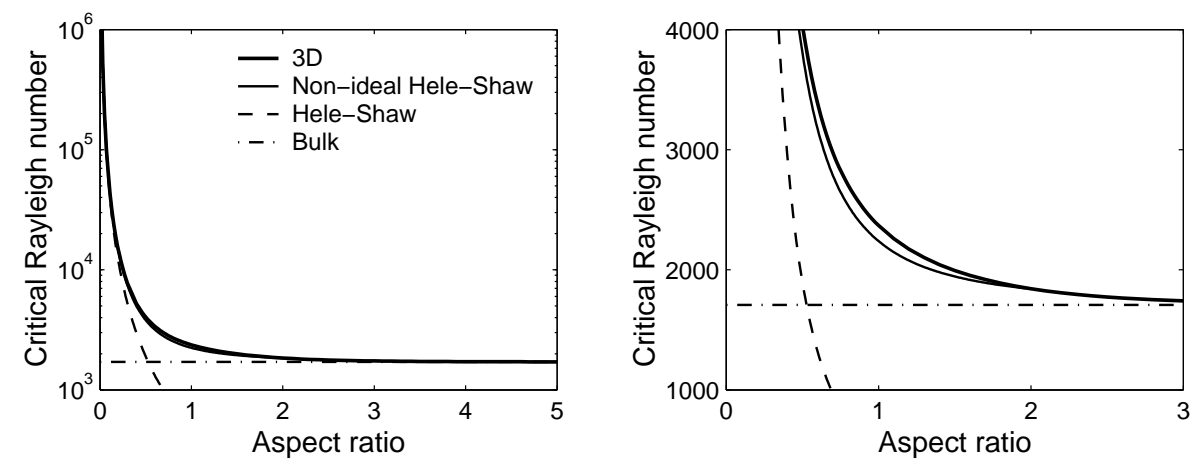

Figure 5. Comparison of the neutral stability curves (critical Rayleigh as a function of the transverse aspect ratio) obtained by solving the 3D stability problem (thick solid line) and by using the non-ideal Hele-Shaw (thin solid line) and the Hele-Shaw (dashed line) approximations for a pure fluid. The dashed-dotted line shows the critical Rayleigh number in the 2D problem.

\section{Conclusions}

In the present paper we have undertaken a numerical study of the linear stability properties of binary fluid mixtures heated from below in three-dimensional containers. We have focused on binary mixtures with negative values of the separation ratio, for which the primary bifurcation is oscillatory. For the first time, we have computed exactly the critical parameters for different values of the transverse aspect ratio of the cell. The comparison of our 3D computations with experiments on a water-ethanol mixture in an annular container (Ohlsen et al., 1990) gives an excellent agreement between the numerical and experimental results.

We have been able to quantify the effect of friction of the transverse walls on the onset of oscillatory binary convection and to check the validity of the approximations usually made to take into account this effect. The results indicate that the presence of the walls has a considerable effect on the critical parameters and that the approximations generally assumed do not reproduce the correct behaviour of the neutral stability curves in a wide range of intermediate transverse aspect ratio cells. The expected delay on the onset of convection due to the presence of the transverse walls is underestimated in the non-ideal Hele-Shaw approximation and the frequency of the arising time-dependent pattern obtained with this model only improves very slightly the value resulting from the $2 \mathrm{D}$ computations for very small aspect ratio cells. We have also found that the neutral stability curves of the $3 \mathrm{D}$ problem showing the dependence of the critical parameters on the

\section{Binary mixture}

\begin{tabular}{|c|c|c|c|c|c|}
\hline$\Gamma_{y}$ & $R a_{c}^{3 D}$ & $R a_{c}^{n i H S}$ & $\left(R a_{c}^{3 D}-R a_{c}^{n i H S}\right) / R a_{c}^{3 D} \cdot 100$ & $R a_{c}^{2 D}$ & $\left(R a_{c}^{3 D}-R a_{c}^{2 D}\right) / R a_{c}^{3 D} \cdot 100$ \\
\hline \hline 3 & 2423 & 2372 & $2.1 \%$ & 2291 & $5.4 \%$ \\
\hline 2 & 2638 & 2473 & $6.3 \%$ & 2291 & $13 \%$ \\
\hline 1 & 3452 & 3014 & $13 \%$ & 2291 & $34 \%$ \\
\hline 0.5 & 5965 & 5151 & $14 \%$ & 2291 & $62 \%$ \\
\hline
\end{tabular}

Pure fluid

\begin{tabular}{|c|c|c|c|c|c|}
\hline$\Gamma_{y}$ & $R a_{c}^{3 D}$ & $R a_{c}^{n i H S}$ & $\left(R a_{c}^{3 D}-R a_{c}^{n i H S}\right) / R a_{c}^{3 D} \cdot 100$ & $R a_{c}^{2 D}$ & $\left(R a_{c}^{3 D}-R a_{c}^{2 D}\right) / R a_{c}^{3 D} \cdot 100$ \\
\hline \hline 2 & 1843 & 1841 & $0.09 \%$ & 1708 & $7.3 \%$ \\
\hline 1 & 2369 & 2239 & $5.5 \%$ & 1708 & $28 \%$ \\
\hline 0.5 & 4041 & 3809 & $5.7 \%$ & 1708 & $58 \%$ \\
\hline
\end{tabular}

Table 2. Numerical values of the critical Rayleigh number for several values of the transverse aspect ratio of the cell for a binary mixture, $S=-0.257$, and for a pure fluid, $S=0$. 

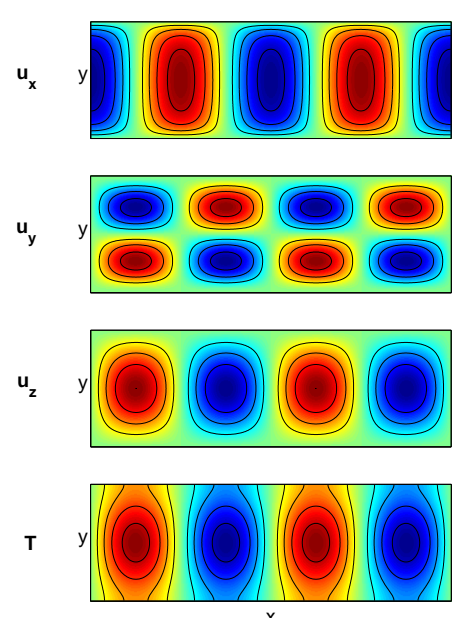
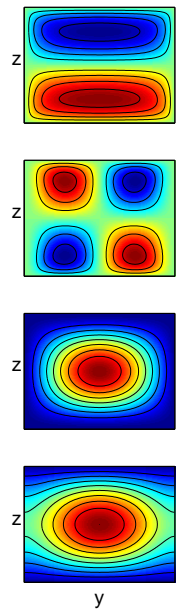
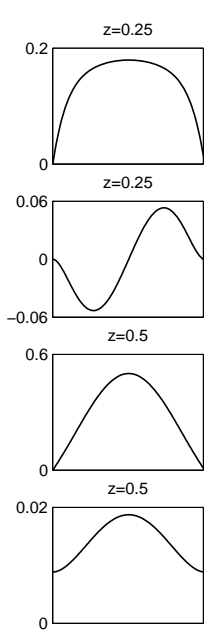

Figure 6. 3D steady-state solution: top and side view. Contour plots of the velocity and temperature fields showing the structure of the 3D steady-state solution for a pure fluid in a vertical and a transversal plane (XY-plane and YZ-plane). Plots of the velocity components and temperature showing their variation with $y$ for a fixed value of $z$.

transverse aspect ratio differ from the 2D results more than might be expected from the results provided by the non-ideal Hele-Shaw approximation.

The origin of these discrepancies needs to be sought in the structure of the time-dependent convective pattern. While the assumptions made in the non-ideal Hele-Shaw approximation (parabolic velocity profile and negligible transverse velocity component) are reasonable in the case of stationary pure fluid convection, we have shown that they are not always justified in the case of the three-dimensional travelling waves arising in binary fluid convection in annular containers. A direct translation of the approximations made in stationary convection to model the effect of the width of the cell does not necessary work well when the flow is oscillatory, as in the case of binary fluid convection.

On the other hand, it is worth mentioning that our code provides very accurate numerical computations of the critical parameters that can be very useful to determine the separation ratio of a binary mixture. The value of the separation ratio is difficult to obtain, and errors of about $30 \%$ are common. An alternative way of determining the separation ratio is to measure first the critical Rayleigh number and frequency experimentally and then to find numerically the value of the separation ratio that reproduces the observed critical parameters (Touiri et al., 1996). In this method, it is obviously important to be able to obtain systematically the exact value of the critical parameters for any set of parameter values that might be relevant to experiments, and this can be achieved with our linear stability code.

In a future work, we plan to analyze the influence of the type of thermal boundary conditions assumed in the transverse walls on the onset of convection. In some experimental setups walls that conduct heat, for which the adiabatic boundary conditions do not fit, are used. We will also study how the codimension 2 point that separates oscillatory from stationary convection in binary mixtures is affected by the presence of the transverse walls. This transition is found to occur in two-dimensional cells for a negative value of the separation ratio slightly smaller than $S=0$ (Knobloch and Moore, 1988). Convection with positive values of the separation ratios will also be considered.

Acknowledgements. We are very grateful to I. Mercader and E. Knobloch for useful discussions. The work of O. Batiste was supported by an EPSRC grant, GR/R52879/01. This work has also been supported by MCYT under grants No. BFM2001-2336 and BFM2002-00670. 


\section{References}

Ohlsen, D.R., Yamamoto, S.Y., Surko, C.M. and Kolodner, P., Transition from traveling-wave to stationary convection in fluid mixtures. Phys. Rev. Lett. 65 (12), (1990) 1431-1434.

Cross, M.C. and Hohenberg, P.C., Pattern formation outside the equilibrium. Rev. Mod. Phys. 65 (3), (1993) 998-1011.

Batiste, O., Net, M., Mercader, I. and Knobloch, E., Oscillatory binary fluid convection in large aspect-ratio containers. Phys. Rev. Lett. 86 (11), (2001) 2309-2312.

Knobloch, E. and Moore, D.R., Linear stability of experimental Soret convection. Phys. Rev. A 37, (1988) 860-870.

Cross, M.C. and Kim, K., Linear stability and the codimension-2 region in binary fluid convection between rigid impermeable boundaries. Phys. Rev. A 37, (1988) 3909-3920.

Batiste, O., Mercader, I., Net, M. and Knobloch, E., Phys. Rev. E 59 (6), (1999) 6730-6741.

Schöpf, W., Convection onset for a binary mixture in a porous medium and in a narrow cell: a comparison. J. Fluid Mech. 245, (1992) 263-278.

Lhost, O. and Platten, J.K., Experimental study of the transition from nonlinear traveling waves to steady overtuning convection in binary mixtures. Phys. Rev. A 40 (8), (1989) 4552-4557.

Kolodner, P., Repeated transients of weakly nonlinear traveling-wave convection. Phys. Rev. E 47 (2), (1993) 1038-1048.

Liu, M. and de Bruyn, J.R., Traveling-wave convection in a narrow rectangular cell. Can. J. Phys. 70, (1992) 689-695.

Schöpf, W. and Rehberg, I., Amplification of thermal noise via convective instability in binary-fluid mixtures. Europhys. Lett. 17 (4), (1992) 321-326.

Bensimon, D., Kolodner, P., Surko, C.M., Williams, H. and Croquette, V., Competing and coexisting dynamical states of travellingwave convection in an annulus. J. Fluid Mech. 217, (1990) 441-467.

Goldstein, H.F., Knobloch, E., Mercader, I. and Net, M., Convection in a rotating cylinder. Part 1. Linear theory for moderate Prandtl numbers. J. Fluid Mech. 28, (1993) 583-604.

Touiri, H., Platten, J.K., and Chavepeyer, G., Effect of the separation ratio on the transition between travelling waves and steady convection in the two-component Rayleigh-Benard problem. Eur. J Mech., B/Fluids 15 (2), (1996) 241-257 